Bull. Austral. Math. Soc.

VoL. 45 (1992) [439-451]

\title{
POSITIVE SOLUTIONS FOR A CLASS OF SEMILINEAR TWO-POINT BOUNDARY VALUE PROBLEMS
}

\author{
Luis Sanchez
}

We study the existence of positive solutions of the periodic, Neumann or Dirichlet problem for the semilinear equation

$$
u^{\prime \prime}+f(t, u)=0, \quad 0 \leqslant t \leqslant T,
$$

where $f$ is a Carathéodory function. Our assumptions in each case are such that the problem possesses a lower solution or an upper solution.

\section{INTRODUCTION}

Let $f:[0, T] \times[0,+\infty) \rightarrow \mathbb{R}$ be a Carathéodory function (that is, measurable in the first variable and continuous in the second one) and consider the differential equation

$$
u^{\prime \prime}+f(t, u)=0 \text {. }
$$

We are concerned with the problem of finding solutions of equation (0) subject to boundary conditions of periodic, Neumann, or Dirichlet type. By definition of $f$, these are nonnegative solutions, that is $u(t) \geqslant 0$ for all $t \in[0, T]$. In some cases we study the special form of $(0)$ in which $f(t, u)=g(u)-h(t)$, where $g:[0,+\infty) \rightarrow \mathbb{R}$ is continuous and $h \in L^{1}(0, T)$. In the general case we assume, without further mention, that $f(t, u)$ has the following property: for each $k>0$ there exists a function $\varphi \in L^{1}(0, T)$ such that, for almost every $t \in[0, T]$ and every $u \in[0, k]$ we have

$$
|f(t, u)| \leqslant \varphi(t) .
$$

Many authors have studied this problem, not only for equation ( 0 ) but also for semilinear elliptic equations in $\mathbb{R}^{N}$. Recent work on the solvability of $(0)$ may be found in the papers of Castro and Shivaj [4], Nkashama and Santanilla [11], Schaaf and Schmitt [16] and references of those papers. As long as the PDE case is concerned we

Received 17 May 1991

The author is indebted to Professor P. Habets and C. de Coster for fruitful discussions that contributed to improvements in some results presented here.

Copyright Clearance Centre, Inc. Serial-fee code: 0004-8729/92 \$A2.00+0.00. 
confine ourselves to draw the attention of the reader to articles by Amann [1], Smoller and Wasserman [17], De Figueiredo [6], Brézis-Oswald [3] and Costa and Goncalves [5].

As is well known, the method of lower and upper solutions yields not only existence of a solution but it also locates the solution between given bounds. To use this method, one must be able to construct a lower solution $\underline{u}$ and an upper solution $\bar{u}$ of $(0)$ (with the appropriate boundary condition) so that $0 \leqslant \underline{u} \leqslant \bar{u}$. The results presented in this paper aim at obtaining existence when a lower solution is given but no upper solution is known, or vice versa, or if a lower solution and an upper solution are given in the wrong order: thus our assumptions will involve the existence of one such lower or upper solution. We shall see that, adding some assumption on the local or asymptotic behaviour of $f(t, u)$, we are still in a position to guarantee, in some instances, the existence of a solution.

\section{PERIOdic SOLUTIONS}

We start by analysing a special form of equation (0), namely

$$
u^{\prime \prime}+g(u)=h(t)
$$

with periodic boundary conditions

$$
u(0)=u(T), \quad u^{\prime}(0)=u^{\prime}(T)
$$

Here, $T>0, h \in L^{1}(0, T)$ and $g:[0, \infty) \rightarrow \mathbb{R}$ is a continuous function.

Let us introduce some notation. The symbol \|\|$_{p}$ will denote the usual norm of $L^{p}(0, T), 1 \leqslant p \leqslant \infty$. For each function $h \in L^{1}(0, T)$, we write $h=\bar{h}+\tilde{h}$, where

$$
\bar{h}=\frac{1}{T} \int_{0}^{T} h(t) d t
$$

so that $\tilde{h}$ has mean value zero on $(0, T)$.

We shall make use of the fixed point index of a compact map in the positive cone of a Banach space (see [1] for instance). Let

$$
C_{+}=\{u \in C[0, T]: u(0)=u(T) \text { and } u(t) \geqslant 0, \quad \forall t \in[0, T]\}
$$

be the positive cone in the space of continuous, $T$-periodic functions. If $\Omega$ is a bounded open set in $C_{+}$, and $F: \bar{\Omega} \rightarrow C_{+}$is a compact mapping such that $F$ has no fixed points on the boundary $\partial_{+} \Omega$ of $\Omega$ relative to $C_{+}$, we denote by $i_{+}(F, \Omega)$ the fixed point index of $F$ in $\Omega$.

In our first results (Theorems 2.1 and 2.2) $u(t) \equiv 0$ is a subsolution of (1)-(2). We first prove two lemmas where a slightly stronger hypothesis, which we call (A.1), is used; this kind of hypothesis appears also in [11]. 
LEMma 2.1. Suppose that there exist $0<a<b$ such that

$$
\begin{gathered}
b-a>\frac{T\|\tilde{h}\|_{1}}{4}, \\
g(u)<\bar{h} \text { if } u \in(a, b),
\end{gathered}
$$

and

(A1) There exists $R>0$ such that for all $0 \leqslant u \leqslant b$ and almost everywhere $t \in[0, t]$, we have

$$
g(u)+R u \geqslant h(t)
$$

Then the problem (1) - (2) has at least one solution $u(t) \geqslant 0$.

REMARK 2.1. Our hypotheses demand, roughly speaking, that either $T$ be small or the interval $(a, b)$ where (4) holds be large. This result is of a kind similar to one of Zanolin [18, Corollary 2].

Proof: Let $g_{0}:[0, \infty) \rightarrow[0,+\infty)$ be defined as $g_{0}(u)=\bar{h}+a-u$ and consider the homotopic equations

$$
\begin{gathered}
u^{\prime \prime}+\lambda g(u)+(1-\lambda) g_{0}(u)=\lambda h+(1-\lambda) \bar{h} \\
u(0)=u(T), u^{\prime}(0)=u^{\prime}(T)
\end{gathered}
$$

and the bounded, open subset of $C_{+}$

$$
\Omega=\left\{u \in C_{+}:\|u\|_{\infty}<b\right\}
$$

We claim that there exist no solutions of (5) on the boundary (relative to $C_{+}$) of $\Omega$, $\partial_{+} \Omega$. To see this we first show that, given a solution $u \in \bar{\Omega}$ of (5) the following estimate holds:

$$
\left|u^{\prime}(t)\right| \leqslant\|\tilde{h}\|_{1} / 2 \text { if } a \leqslant u(t) \leqslant b .
$$

In order to prove (6) we remark that integrating (5) in $[0, T]$ and using (4) we conclude that for some $s \in[0, T]$ we have $u(s)<a$. Now let $t_{0} \in \mathbb{R}$ be such that $a \leqslant u\left(t_{0}\right) \leqslant b$ and $u^{\prime}\left(t_{0}\right)>0$, for a given solution $u \in \bar{\Omega}$. Extending $u$ to $\mathbb{R}$ as a $T$-periodic function and using the above remark we may choose $t_{1}>t_{0}$ such that $a \leqslant u(t) \leqslant b$ if $t_{0} \leqslant t \leqslant t_{1}$ and $u^{\prime}\left(t_{1}\right)=0$. Then (5) yields

$$
-u^{\prime}\left(t_{0}\right)+\int_{t_{0}}^{t_{1}}\left(g_{\lambda}(u)-\bar{h}\right) d t=\lambda \int_{t_{0}}^{t_{1}} \tilde{h} d t
$$


where we have set $g_{\lambda}(u)=\lambda g(u)+(1-\lambda) g_{0}(u)$. The integrand in the left-hand side is negative because of (4), so that

$$
-u^{\prime}\left(t_{0}\right) \geqslant-\lambda \int_{t_{0}}^{t_{1}} \tilde{h}^{-} d t \geqslant-\|\tilde{h}\|_{1} / 2
$$

and (6) holds. A similar argument applies if $u^{\prime}\left(t_{0}\right)<0$.

Now let $u \in \partial_{+} \Omega$ be a solution of (5). Then $\|u\|_{\infty}=b$ and we may choose $t_{1}<t_{2}<t_{3}$ such that $u\left(t_{1}\right)=u\left(t_{3}\right)=a, u\left(t_{2}\right)=b$ and $a \leqslant u(t) \leqslant b$ if $t_{1} \leqslant t \leqslant t_{3}$. Using (6) we deduce that

$$
\begin{aligned}
& b-a=u\left(t_{2}\right)-u\left(t_{1}\right) \leqslant\left(t_{2}-t_{1}\right)\|\tilde{h}\|_{1} / 2, \\
& b-a=u\left(t_{2}\right)-u\left(t_{3}\right) \leqslant\left(t_{3}-t_{2}\right)\|\tilde{h}\|_{1} / 2,
\end{aligned}
$$

and it follows that

$$
2(b-a) \leqslant T\|\tilde{h}\|_{1} / 2,
$$

a contradiction with (3). Thus our claim is proved.

Denote by $K: L^{1}(0, T) \rightarrow W^{2,1}(0, T)$ the inverse of the linear differential operator $-u^{\prime \prime}+R u$ with periodic conditions (2). We take $R$ in (A1) so large that also $g_{0}(u)+$ $R u \geqslant \bar{h}$ whenever $u \geqslant 0$. Let

$$
N(\lambda, u)=g_{\lambda}(u)+R u-\lambda h-(1-\lambda) \bar{h} .
$$

Then $N$ is a continuous mapping of $[0,1] \times \bar{\Omega}$ into the positive cone of $L^{1}(0, T)$; it takes bounded sets into bounded sets. Since $K$ is a positive linear operator, the product $K N:[0,1] \times \bar{\Omega} \rightarrow C_{+}$is compact and we see that (5) may be written simply as

$$
u=K N(\lambda, u), \quad u \in \bar{\Omega} \text {. }
$$

From what we have proved above and the homotopy invariance of the fixed point index we get

$$
i_{+}(K N(1, .), \Omega)=i_{+}(K N(0, .), \Omega) \text {. }
$$

When $\lambda=0$, the only solution of (7) is $u \equiv a \in \Omega$ as (5) shows. By linearisation we easily obtain

$$
i_{+}(K N(0, .), \Omega)=1 \text {. }
$$

Therefore (8) and the existence property of the fixed point index implies that (7) is solvable in $\Omega$ for $\lambda=1$ as well.

LEMMA 2.2. Suppose that there exist $0<a<b$ such that (A1), (4) are satisfied and

$$
\int_{a}^{b}(g(u)-\bar{h}) d u<-\frac{\|\tilde{h}\|_{1}^{2}}{2}
$$

Then problem (1) - (2) has at least one solution $u \geqslant 0$. 
REMARK 2.2. Unlike condition (3), (9) holds if $\|\tilde{h}\|_{1}$ is sufficiently small (regardless of the period).

Proof: Take a function $g_{0}$ of the form $g_{0}(u)=\bar{h}+k(a-u)$ where $k>\|\tilde{h}\|_{1}^{2}$. $(b-a)^{-2}$, so that, for any $\lambda \in[0,1]$, we have

$$
\int_{a}^{b}\left(g_{\lambda}(u)-\bar{h}\right) d u<-\frac{\|\tilde{h}\|_{1}^{2}}{2}
$$

where $g_{\lambda}=\lambda g+(1-\lambda) g_{0}$. Consider $\Omega$ and the homotopy (5) as in the proof of Lemma 2.1. Let us show that (5) has no solution in $\partial_{+} \Omega$. For, if $u$ is such a solution, we may choose $t_{1}<t_{2}$ such that $u\left(t_{1}\right)=a, u\left(t_{2}\right)=b, a \leqslant u(t) \leqslant b$ if $t_{1} \leqslant t \leqslant t_{2}$, and then multiplying (5) by $u^{\prime}$ and integrating we have

$$
-\frac{u^{\prime}\left(t_{1}\right)^{2}}{2}+\int_{a}^{b}\left(g_{\lambda}(u)-\bar{h}\right) d u=\lambda \int_{t_{1}}^{t_{2}} \tilde{h} u^{\prime} d t .
$$

Using the estimate (6) we obtain:

$$
\int_{a}^{b}\left(g_{\lambda}(u)-\bar{h}\right) d u \geqslant-\int_{t_{1}}^{t_{2}}\left|\widetilde{h} u^{\prime}\right| d t \geqslant-\frac{\|\widetilde{h}\|_{1}^{2}}{2}
$$

a contradiction with (10). Hence we compute, as in the preceeding lemma,

$$
i_{+}(K N(0, .), \Omega)=1
$$

and the proof is complete.

THEOREM 2.1. Suppose that $g(0) \geqslant h(t)$ for almost every $t \in[0, T]$ and there exist $0<a<b$ satisfying (3) and (4). Then the problem (1)-(2) has at least one solution $u(t) \geqslant 0$.

Proof: Let $\varepsilon>0$ and consider the perturbed equation

$$
u^{\prime \prime}+g(u)=h(t)-\varepsilon
$$

Choose $a<a^{\prime}<b^{\prime}<b$ so that $b^{\prime}-a^{\prime}>T\|\tilde{h}\|_{1} / 4$. Then if $\varepsilon$ is sufficiently small all the assumptions of Lemma 2.1 are satisfied with respect to $(1)_{e}-(2)$. Lemma 2.1 implies that $(1)_{e}-(2)$ has a solution $u_{e}(t)$ such that $0 \leqslant u_{\varepsilon}(t) \leqslant b$. A standard argument shows that the family $\left(u_{e}\right)$ is (bounded and) equicontinuous in $C[0, T]$. Passing to the limit along a convenient subsequence as $\varepsilon \rightarrow 0$ yields the result.

Using Lemma 2.2 and a similar approximation argument, one proves: 
Theorem 2.2. Suppose that $g(0) \geqslant h(t)$ for almost every $t \in[0, T]$ and there exist $0<a<b$ such that (4) and (9) are satisfied. Then problem (1)-(2) has at least one solution $u(t) \geqslant 0$.

In our next theorem the assumptions imply in particular that we have a lower solution $\bar{u}(t) \equiv a>0$ and an upper solution $\underline{u}(t) \equiv 0$ (thus in the wrong order) for problem (0)-(2). Precisely, let us state (see [11]):

(A2) There exists $R \in\left(0, \pi^{2} T^{-2}\right]$ such that $f(t, u) \leqslant R u$ for all $t \in[0, T]$ and $u \geqslant 0$.

The significance of the bound for $R$ in (A2) is the following. If $0<R \leqslant \pi^{2} / T^{2}$, then the (unique) solution of

$$
\begin{gathered}
u^{\prime \prime}+R u=h(t) \\
u(0)=u(T), u^{\prime}(0)=u^{\prime}(T)
\end{gathered}
$$

where $h \in L^{1}(0, T)$ and $h$ is nonnegative, is itself nonnegative. In fact, multiplying (11) by $\cos \sqrt{R}\left(t-t_{0}\right)$, then by $\sin \sqrt{R}\left(t-t_{0}\right)$ and integrating over $\left[t_{0}, t_{0}+T\right]$ (we assume that $h(t)$ is $T$-periodically extended) we are left with a linear system which yields

$$
u\left(t_{0}\right)=\frac{\int_{t_{0}}^{t_{0}+T} h(t)\left[\sin \sqrt{R}\left(t-t_{0}\right)+\sin \sqrt{R} T-\left(t-t_{0}\right)\right] d t}{2 \sqrt{R}(1-\cos \sqrt{R} T)}
$$

and the remark easily follows.

THEOREM 2.3. Let $f(t, u)$ satisfy (A2). Assume also that there exist $a>0$ and $\varepsilon>0$ such that

$$
f(t, u) \geqslant 0, \text { for all } u \in[a, a+\varepsilon] \text { and almost every } t \in[0, T]
$$

and either $R<2 T^{-2}$ or there exists $\alpha \in L^{1}(0, T)$ such that, for $t \in[0, T]$ and $u \geqslant 0$,

$$
f(t, u) \geqslant \alpha(t) \text {. }
$$

Then problem (0)-(2) has at least one solution $u \geqslant 0$.

Proof: Choose $a^{\prime}<a$, close to $a$. Consider the homotopic equations

$$
\begin{gathered}
u^{\prime \prime}+\lambda f(t, u)+(1-\lambda) \sigma\left(u-a^{\prime}\right)=0 \\
u(0)=u(T), u^{\prime}(0)=u^{\prime}(T)
\end{gathered}
$$

where $\sigma \in(0, R)$ and $0 \leqslant \lambda \leqslant 1$. We claim that there exists $A>0$ such that, if $u(t)$ is a solution of $(14)$ for some $\lambda \in[0,1]$ and $\min u \leqslant a$, then

$$
u(t)<A \text { for all } t \in[0, T] \text {. }
$$


To prove this, assume first that $R<2 T^{-2}$. Using Proposition 3.1 in [8] we obtain for solutions of (14) the inequality

$$
\left\|u^{\prime}\right\|_{\infty} \leqslant \int_{0}^{T}\left[\lambda f(t, u)+(1-\lambda) \sigma\left(u-a^{\prime}\right)\right]^{+} d t \leqslant R \int_{0}^{T} u d t .
$$

If we choose $t_{m} \in[0, T]$ such that $u\left(t_{m}\right) \leqslant a$, we have

$$
\left\|u^{\prime}\right\|_{\infty} \leqslant R T a+R \int_{0}^{T} d t \int_{t_{m}}^{t} u^{\prime}(s) d s \leqslant R T a+\frac{R T^{2}}{2}\left\|u^{\prime}\right\|_{\infty}
$$

so that $\left\|u^{\prime}\right\|_{\infty} \leqslant 2 R T a\left(2-R T^{2}\right)^{-1}$. Therefore (15) is satisfied with $A=a+$ $2 R T^{2} a\left(2-R T^{2}\right)^{-1}+1$. Next assume (13). Then we obtain the estimate

$$
\left\|u^{\prime}\right\|_{\infty} \leqslant \int_{0}^{T}\left[\lambda f(t, u)+(1-\lambda) \sigma\left(u-a^{\prime}\right)\right]^{-} d t \leqslant\|\alpha\|_{1}+\sigma a^{\prime} T
$$

so that (15) holds with $A=a+T\left(\|\alpha\|_{1}+\sigma a T\right)+1$.

Now we take the bounded, open set

$$
\Omega=\left\{u \in C_{+}: \min u<a,\|u\|_{\infty}<A\right\} .
$$

From what has been proved above we can assert that, if $0 \leqslant \lambda<1$, (14) has no solution in $\partial_{+} \Omega$. In fact the possibility that $\min u=a$ for such a solution $u(t)$ is ruled out by (12). Otherwise we would be able to choose an interval $\left[t_{0}, t_{1}\right]$ such that $u\left(t_{0}\right)=a$, $u^{\prime}\left(t_{1}\right) \geqslant 0, u(t) \leqslant a+\varepsilon$ if $t \in\left[t_{0}, t_{1}\right]$ and (14) would imply

$$
0=u^{\prime}\left(t_{1}\right)+\int_{t_{0}}^{t_{1}}\left[\lambda f(t, u)+(1-\lambda) \sigma\left(u-a^{\prime}\right)\right] d t>0
$$

a contradiction. Rewriting (14) as

$$
u=S\left[R u-\lambda f(t, u)-(1-\lambda) \sigma\left(u-a^{\prime}\right)\right]
$$

where $S$ is the inverse of the linear operator $u^{\prime \prime}+R u$ with periodic conditions (which, as the remark preceeding the theorem shows, sends nonnegative functions into $C_{+}$), we conclude: either (0)-(2) has a solution $u \in \bar{\Omega}$ or $i_{+}(S N(1,),. \Omega)=1$, where

$$
N(\lambda, u)=R u-\lambda f(t, u)-(1-\lambda) \sigma\left(u-a^{\prime}\right),
$$

in which case (0)-(2) has a solution $u \in \Omega$. This ends the proof. 
REMARK 2.3. Assuming that $f$ is continuous in $[0, T] \times \mathbb{R}_{+}$, it is easily seen that the proof works (even in a simpler form) if (12) is stated simply as

$$
f(t, a) \geqslant 0, \quad t \in[0, T] .
$$

In the next theorem we return to equation (1), and $u(t) \equiv 0$ is again a subsolution.

THEOREM 2.4. Suppose that

$$
g(0) \geqslant h(t) \text { for almost every } t \in[0, T],
$$

that $L:=\lim _{u \rightarrow+\infty}(h u-G(u))$ exists, where $G(u)=\int_{0}^{u} g(s) d s,(u \geqslant 0)$, and for some $R>0$ we have

$$
\bar{h} u-G(u) \leqslant L \quad \text { if } u \geqslant R .
$$

Then problem (1)-(2) has at least one solution $u \geqslant 0$.

Proof: Let us extend $g$ to $(-\infty, 0]$, defining $g(u)=g(0)$ if $u<0$ and let us still denote by $G(u)$ the primitive of the extended function. Consider the $C^{1}$ functional

$$
\begin{aligned}
J(u) & =\int_{0}^{T}\left[\frac{u^{\prime 2}}{2}+h(t) u-G(u)\right] d t \\
& =\int_{0}^{T}\left[\frac{u^{\prime 2}}{2}+(h u-G(u))+\widetilde{h}(t) u\right] d t
\end{aligned}
$$

defined in the Sobolev space $H_{T}^{1}=\left\{u \in H^{1}(0, T): u(0)=u(T)\right\}$. It is easily seen that the method used in [14, Theorem 1] or [15, Theorem 1] may be adapted to show that $J$ attains a minimum in $B_{T}^{1}$ : it is enough to check that (i) the function $\bar{h} u-G(u)$ is bounded below, and (ii) $\lim _{u \rightarrow-\infty} \bar{h} u-G(u):=L^{\prime}$ exists and $\bar{h} u-G(u) \leqslant L^{\prime}$ if $u \leqslant 0$. Now (ii) follows from (16) and the fact that $\bar{h} u-G(u)=(\bar{h}-g(0)) u$ if $u \leqslant 0$. For the same reason we have $L^{\prime}=0$ or $L^{\prime}=+\infty$; also $L>-\infty$ on account of (17), and (i) holds. Hence $J$ has indeed a minimum attained at some function $u(t)$ which solves (1)-(2) with the extended function $g$. It remains to show that $u(t) \geqslant 0$ for all $t \in[0, T]$. This is a straightforward consequence of $(16)$ and the definition of $g(u)$ for $u<0$.

REMARK 2.4. Theorems $2.1,2.2$ and 2.4 extend naturally to the case where one considers the Neumann boundary condition $u^{\prime}(0)=0, u^{\prime}(T)=0$. As long as Theorem 2.3 is concerned, the only difference is that in the assumption A2 one should write

$$
0<R \leqslant \pi^{2} /\left(4 T^{2}\right)
$$

(see [7]). 


\section{DiRICHLET BOUNDARY CONDITIONS}

In this section we consider the boundary value problem

$$
\begin{gathered}
u^{\prime \prime}+f(t, u)=0 \\
u(0)=0, \quad u(\pi)=0
\end{gathered}
$$

where the Carathéodory function $f$ is defined in $[0, \pi] \times \mathbb{R}_{+}$and is such that, for each $K>0$, there exists a function $\alpha \in L^{1}(0, \pi)$ such that $|f(t, u)| \leqslant \alpha(t)$ if $t \in[0, \pi]$ and $0 \leqslant u \leqslant K$. To motivate our setting of the problem some remarks are in order. Let $m \in L^{\infty}(0, \pi)$ be a function such that $m(t)>0$ in a set of positive measure and denote by $\mu(m)$ the first positive eigenvalue of the linear problem (see [10])

$$
\begin{aligned}
& u^{\prime \prime}+\lambda m(t) u=0, \\
& u(0)=0, u(\pi)=0 .
\end{aligned}
$$

Then, if

$$
\begin{gathered}
\underset{u \rightarrow 0^{+}}{\liminf } \frac{f(t, u)}{u}=a(t), \quad \underset{u \rightarrow+\infty}{\limsup } \frac{f(t, u)}{u}=b(t) \\
\mu(a)<1<\mu(b),
\end{gathered}
$$

and

we can construct a lower solution $\underline{u}>0$ and an upper solution $\bar{u}$ of (18)-(19), such that $\underline{u} \leqslant \bar{u}$ (see [5]) or else we can solve the problem through minimisation of the associated functional, see [3]. A quite different situation occurs if

and

$$
\underset{u \rightarrow 0^{+}}{\limsup } \frac{f(t, u)}{u}=a(t), \quad \liminf _{u \rightarrow+\infty} \frac{f(t, u)}{u}=b(t)
$$

$$
\mu(a)>1>\mu(b)
$$

this may be studied through the fixed-point index (see [1]) or the time map (see [12]). Here we are interested in starting from an hypothesis similar to this one but only where the behaviour near zero is concerned; we then add a one-sided Landsman-Lazer condition. Note that if $\mu(a)>1$ holds as above, it is easy to see that (18)-(19) has a (small) positive upper solution. Precisely, we state:

(A3) Let $F(t, u)=\int_{0}^{u} f(t, s) d s$. We assume that $f(t, 0)=0$ almost everywhere and that there exist $\varepsilon>0$ and a function $a \in L^{\infty}(0, \pi), a(t) \geqslant 0$ almost everywhere, such that for almost every $t \in[0, \pi]$ and $0 \leqslant u \leqslant \varepsilon$, we have

$$
F(t, u) \leqslant a(t) u^{2} / 2
$$


and $\mu(a)>1$.

For convenience we now write $f(t, u)=u+p(t, u)$ and accordingly (18) turns into

$$
u^{\prime \prime}+u+p(t, u)=0 \text {. }
$$

It is easily seen that (A3) implies that $p(t, u)$ is negative somewhere to the right of zero, for small $u$.

THEOREM 3.1. Let $f$ satisfy (A3) and, with the notation introduced in $\left(18^{\prime}\right)$, assume that there exists a function $\beta \in L^{1}(0, \pi)$ such that, for almost every $t \in[0, T]$ and $u \geqslant 0$,

$$
|p(t, u)| \leqslant \beta(t)
$$

moreover let $p$ satisfy the Landesman-Lazer condition

$$
\int_{0}^{\pi} p_{+}(t) \sin t d t>0
$$

where $p_{+}(t)=\liminf _{u \rightarrow+\infty} p(t, u)$. Then (18)-(19) has a (nontrivial) nonnegative solution.

Proof: Extend $f(t, u)$ to all values of $u \in \mathbb{R}$ by setting $f(t, u)=0$ if $u \leqslant 0$. For simplicity, we denote by the same symbol the corresponding extensions of $p(t, u)$, $F(t, u)$. Let $P(t, u)=\int_{0}^{u} p(t, s) d s$. We consider the functional

$$
J(u)=\int_{0}^{\pi}\left[\frac{u^{\prime 2}}{2}-\frac{u^{2}}{2}-P(t, u)\right] d t
$$

which is of class $C^{1}$ in $H_{0}^{1}(0, \pi)$, and we look for a critical point $u \neq 0$ of $J$. To prove that such a critical point exists we use the mountain-pass lemma [2].

Step 1. $J$ has a strict local minimum at the origin. This is an easy consequence of the injection $H_{0}^{1}(0, \pi) \subset C[0, \pi]$ which, combined with the fact that (A3) obviously holds for $|u| \leqslant \varepsilon$ with respect to the extended function $F(t, u)$, shows that, for some $\delta>0$, $\|u\|<\delta$ (where $\|u\|$ is a norm of $u$ in $H_{0}^{1}(0, \pi)$ ) implies

$$
J(u) \geqslant \int_{0}^{\pi}\left(\frac{u^{\prime 2}}{2}-a(t) \frac{u^{2}}{2}\right) d t .
$$

Since $\mu(a)>1$, the quadratic form in the right-hand side is positive definite in $H_{0}^{1}(0, \pi)$. In particular we can fix $\delta>0$ and $c>0$ such that

$$
J(u) \geqslant c \quad \text { if } \quad\|u\|=\delta .
$$


STEP 2. There exists $v \in H_{0}^{1}(0, \pi)$, with $\|v\|$ large, such that $J(v) \leqslant 0$. In fact, it can be shown as in $[9$, p.39] or $[14$, Theorem 4.1] that (21) implies

$$
\lim _{b \rightarrow+\infty} J(b \sin t)=-\infty
$$

STEP 3. $J$ satisfies the Palais-Smale condition. Indeed let $u_{n} \in H_{0}^{1}(0, \pi)$ and $M \in \mathbb{R}$ be such that

$$
J\left(u_{n}\right) \leqslant M, \quad J^{\prime}\left(u_{n}\right) \rightarrow 0 .
$$

It suffices to show that $\left(u_{n}\right)$ is bounded since the remaining properties of $\left(u_{n}\right)$ follow in a standard way, see [13]. We have, because of (20),

$$
\begin{aligned}
\int_{0}^{\pi} \frac{u_{n}^{\prime 2}}{2} d t & \leqslant \int_{u_{n}>0} F\left(t, u_{n}\right) d t+M \\
& =\int_{u_{n}>0} \frac{u_{n}^{2}}{2} d t+\int_{u_{n}>0} P\left(t, u_{n}\right) d t+M \\
& \leqslant \int_{0}^{\pi} \frac{u_{n}^{2}}{2} d t+c_{1}\left\|u^{+}\right\|_{\infty}+M
\end{aligned}
$$

where $c_{1}=\|\beta\|_{1}$. Splitting $u_{n}$ as usual into $u_{n}=a_{n} \sin t+w_{n}$, $\left(a_{n} \in \mathbb{R}, \int_{0}^{\pi} w_{n}(t) \sin t d t=0\right)$, and letting $c_{2}, c_{3}, c_{4}$ denote positive constants independent of $n$,

$$
\begin{gathered}
\int_{0}^{\pi}\left(\frac{w_{n}^{\prime 2}}{2}-\frac{w_{n}^{2}}{2}\right) d t \leqslant c_{2}\left(\left|a_{n}\right|+\left\|w_{n}\right\|\right)+M \\
\left\|w_{n}\right\|^{2} \leqslant c_{3}\left(\left|a_{n}\right|+\left\|w_{n}\right\|\right)+c_{4} .
\end{gathered}
$$

Now we argue by contradiction: suppose that $\left|a_{n}\right| \rightarrow \infty$ (at least along some subsequence). Then from (23) easily follows, as in the proof of Theorem 4.1 in [14], that

$$
v_{n}=\frac{w_{n}}{a_{n}} \rightarrow 0 \text { in } H_{0}^{1}(0, \pi) \text { and uniformly in }[0, \pi]
$$

We must examine two possible cases: (i) $a_{n} \rightarrow+\infty$, and (ii) $a_{n} \rightarrow-\infty$. Since $u_{n}=a_{n}\left(\sin t+v_{n}\right)$, we have $u_{n}(t) \rightarrow+\infty$ if $t \in(0, \pi)$ in case (i). Since $p(t, u)=-u$ if $u<0$ and (20) holds, the sequence $p\left(t, u_{n}(t)\right)$ is bounded below by an integrable function, and Fatou's lemma implies

$$
\int_{0}^{\pi} \liminf _{n \rightarrow \infty} p\left(t, u_{n}(t)\right) \sin t d t \leqslant \lim \int_{0}^{\pi} p\left(t, u_{n}(t)\right) \sin t d t=0
$$


where the last equality comes from

$$
\left\langle J^{\prime}\left(u_{n}\right), \sin t\right\rangle \rightarrow 0
$$

Since $p_{+}(t) \leqslant \lim$ inf $p\left(t, u_{n}(t)\right)$, we have reached a contradiction with (21). In case (ii) we may choose $N \in \mathbb{N}$ such that if $n \geqslant N, u_{n}(t)<0$ in $[\pi / 3,2 \pi / 3]=I$. Using the decomposition, for $n \geqslant \mathbb{N}$,

$$
\int_{0}^{\pi} p\left(t, u_{n}(t)\right) \sin t d t=\int_{I}\left|u_{n}(t)\right| \sin t d t+\int_{[0, \pi] \backslash I} p\left(t, u_{n}(t)\right) \sin t d t
$$

and noting that the integrand in the last integral is bounded below, we conclude that

$$
\lim \int_{0}^{\pi} p\left(t, u_{n}(t)\right) \sin t d t=+\infty
$$

again a contradiction. This ends the proof of Step 3.

From Steps 1 to 3 we conclude that $J$ has a critical value $\geqslant c$. In particular the corresponding critical point is a nonzero function $u(t)$. This function is a solution of (18)-(19) with the extended function. But then an elementary version of the maximum principle implies that $u(t) \geqslant 0$ for all $t \in[0, T]$, and the proof of the theorem is complete.

REMARK 3.1. It is easy to see that the same proof works, as in [14], with (20) replaced for the less restrictive hypothesis:

$$
\limsup _{u \rightarrow+\infty} \frac{P(t, u)}{u^{2}}=0
$$

\section{References}

[1] H. Amann, 'Fixed point equations and nonlinear eignevalue problems in ordered Banach spaces', SIAM Review 18 (1976), 620-709.

[2] A. Ambrosetti and P.H. Rabinowitz, 'Dual variational methods in critical point theory and applications', J. Funct. Anal. 14 (1973), 349-381.

[3] H. Brézis and L. Oswald, 'Remarks on sublinear elliptic equations', Nonlinear Anal. T.M.A. 10 (1986), 55-64.

[4] A. Castro and R. Shivaji, 'Non-negative solutions for a class of non-positone problems', Proc. Roy. Soc. Edinburgh 108A (1988), 291-302.

[5] D. Costa and J.V. Gonçalves, ' $O$ n the existence of positive solutions for a class of non-selfadjoint elliptic boundary value problems', Appl. Anal. 31 (1989), 309-320.

[6] D.G. Figueiredo, 'Positive solutions for some classes of semilinear elliptic problems', in Proc. Symp. Pure Mathematics 45, Part 1, Editor F. Browder, pp. 371-379, 1986. 
[7] P. Habets, M. Ramos and L. Sanchez, 'Jumping non-linearity for $2^{\text {nd }}$ order ODE with positive forcing', in Delay Differential Equations and Dynamical Systems: Lecture Notes in Mathematics 1475, Proceedings Claremont 1990, Editors S. Busenbert and M. Martelli, pp. 191-203, 1991 .

[8] A.C. Lazer and S. Solimini, 'On periodic solutions of nonlinear differential equations with singularities', Proc. Amer. Math. Soc. 99 (1987), 109-114.

[9] J. Mawhin, 'Problèmes de Dirichlet variationels non linéaires', in Séminaire de Mathématiques Supérieures 104 (Les Presses de l'Université de Montréal, 1987).

[10] A. Manes and A.M. Micheletti, 'Un'estensione della teoria variazionale classica degli autovalori per opperatori ellittici del secondo ordine', Boll. Un. Mat. Ital. (4) 7 (1973), 285-301.

[11] M.N. Nkashama and J. Santanilla, 'Existence of multiple solutions for some nonlinear boundary value problems', J. Differential Equations 84 (1990), 148-164.

[12] Njoku and F. Zanolin, 'Positive solutions for two-point BVP's: existence and multiplicity results', Nonlinear Anal. T.M.A. 13 (1989), 1329-1338.

[13] P.H. Rabinowitz, 'Minimax methods in critical point theory with applications to differential equations', in CBMS Reg. Conf. Ser. in Math. 65 (Amer. Math. Soc., Providence RI, 1986).

[14] M. Ramos and L. Sanchez, 'Variational elliptic problems involving noncoercive functionals', Proc. Roy. Soc. Edinburgh 112A (1989), 177-185.

[15] L. Sanchez, 'Boundary value problems for some fourth order ordinary differential equations', Appl. Anal. 38 (1990), 161-177.

[16] R. Schaaf and K. Schmitt, 'A class of nonlinear Sturm-Liouville problems with infinitely many solutions', Trans. Amer. Math. Soc. 306 (1988), 853-859.

[17] J. Smoller and A. Wasserman, 'Existence of positive solutions for semilinear elliptic equations in general domains', Arch. Rational Mech. Anal. 95 (1986), 217-225.

[18] F. Zanolin, 'Remarks on multiple periodic solutions for non-linear ordinary differential systems of Liénard type', Boll. Un. Mat. Ital. (6) 1-B (1982), 683-698.

Instituto Nacional de Investigacao Cientifica Centro de Matematica e Aplicacoes Fundamentais 1699 Lisboa Codex

Portugal 